\title{
Pediatric Ventriculoperitoneal Shunt: Outcome Analysis in a Tertiary Care Center in Nepal
}

\author{
Suman Phuyal, Binod Rajbhandari, Amit B Pradhanang, Gopal Sedain, Sushil K Shilpakar, Mohan R \\ Sharma \\ Department of Neurosurgery, Maharajgunj Medical Campus, Tribhuvan University Teaching Hospital, Institute of \\ Medicine, Maharajgunj, Kathmandu, Nepal
}

\section{Corresponding author:}

Suman Phuyal, MBBS, MS

Department of Neurosurgery, Maharajgunj Medical Campus, Tribhuvan University Teaching Hospital, Institute of Medicine, Maharajgunj, Kathmandu, Nepal

Email:suman42phuyal@gmail.com

Submitted : Mar 1, 2020

Accepted : Apr 4, 2020

\begin{abstract}
Introduction

Ventriculoperitoneal (VP) shunt is a commonly performed neurosurgical procedure in pediatric surgical practice. It results in a dramatic improvement in patient survival and neurological function but is associated with several complications requiring multiple shunt revisions throughout a patient's lifetime. The main aim of the study was to evaluate the outcome especially complications of shunt surgery at our center.

\section{Methods}

A single-institutional, retrospective observational study was conducted in 60 operated cases of congenital hydrocephalus with a minimum follow-up for up to 6 months. Parameters for analysis included demographic data, etiology of hydrocephalus, surgery time, and type of complications.

\section{Results}

Out of 86 patients, 60 patients who fulfilled the inclusion criteria were enrolled in the study. The mean age of the patients at the time of VP shunt placement was 3 months, range from 8 days to 15 years with male preponderance. The majority of the patients belonged to the 0 -1-year age group (76.8\%). The commonest etiology of hydrocephalus was congenital aqueduct stenosis. Fifteen patients (25\%) developed complications within six months follow-up period. Eight patients (13.3\%) developed clinical shunt infection with four cases having culture positive. Four (6.67\%) had mechanical complications in the form of shunt obstruction. Three patients had ascites, subdural collection, and shunt extrusion from the anus. The shunt related mortality was $1.67 \%$.
\end{abstract}

\section{Conclusion}

The shunt infection and obstruction still remain the most important complications. Despite inherent problems, shunt surgery is the procedure of choice until a safer effective alternative is available.

Keywords: Complications congenital hydrocephalus, ventriculoperitoneal shunt 


\section{INTRODUCTION}

$\mathrm{H}$ ydrocephalus (HCP) is an active distension of the ventricular system of the brain, resulting from the inadequate passage of cerebrospinal fluid from its point of production within the cerebral ventricles to its point of absorption into the systemic circulation. ${ }^{1}$ It is a common condition of childhood, and associated with numerous other diseases, including spina bifida and other congenital anomalies, brain tumors, head injury, brain hemorrhage, and meningitis. The incidence of congenital HCP is highest in Latin America which is 316 per 100,000 births and lowest in US/ Canada which is 68 per 100,000 births. ${ }^{2}$ Similarly, the incidence in South-East Asia Region is 76 per 100,000 births. $^{2}$

Treatment of hydrocephalus with ventriculoperitoneal (VP) shunt is an established procedure which was first performed in 1908 by Walther Kausch who used a rubber conduit to drain the lateral ventricle into the peritoneal cavity. ${ }^{3}$ Shunt placement for hydrocephalus accounted for $14 \%$ of caseload in a tertiary neurosurgical center. ${ }^{4}$ Shuntrelated complications constitutes a significant health care burden. Despite several modifications, it is associated with many complications and may require multiple surgical procedures during a patient's life span. ${ }^{5}$

Complications of VP shunt surgery may be broadly divided into (1) mechanical and (2) infective complications. Mechanical complications include obstruction, disconnection, or migration of a shunt system either at the ventricular or peritoneal end. Infective complications such as ventriculitis, shunt tract abscess, and skin necrosis overlying the shunt device. Other complications include subdural collection, inguinal hernia, hydrocele, ascites, pseudocyst formation, perforation of a viscus, or extrusion of the shunt. ${ }^{6}$ Despite its complications, VP shunt surgery is still commonly performed to treat HCP in developing countries like Nepal. There are only a few studies performed to study VP shunt complications and no study till date done at our center to evaluate its outcome especially complications of shunt surgery.

\section{METHODS}

This is a retrospective observational study that included VP shunt surgeries conducted over a duration of one year, from January 2018 to December 2018. Ethical clearance from the Institutional Review Committee was obtained. Only the pediatric population up to the age of 16 years with a diagnosis of acute HCP was included in this study which was conducted in the Department of Neurosurgery, Tribhuvan University Teaching Hospital, Kathmandu, Nepal.

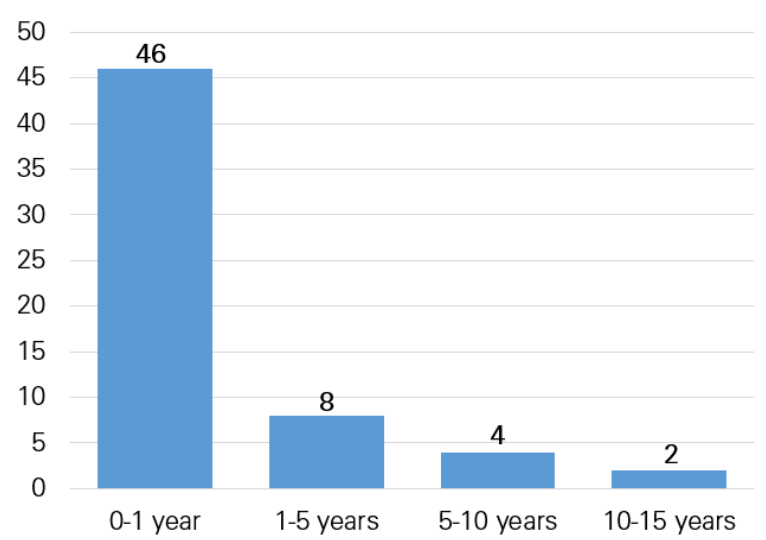

Fig 1. Age at shunt placement

The children undergoing a first-time shunt procedure during the study period were included; and the children who underwent shunt revisions, external ventricular drain, and lumboperitoneal shunts were excluded. All patients were either examined in the Emergency Room or the Neurosurgical Out-patient Clinic. All patients had undergone a preoperative computerized tomography (CT) scan.

A database was created using Microsoft Excel that includes patient demographics, etiology of HCP, date and duration of surgery, type of complications, as well as follow-up reviews for up to six months. All data were converted into IBM SPSS Statistics for statistical analysis. Data were summarized using frequency distribution tables and graphical methods.

\section{RESULTS}

A total of 86 patients who had undergone VP shunt admitted in the Department of Neurosurgery over a period of one year were evaluated. Sixty patient fulfilled inclusion criteria were enrolled in the study and were analyzed within a follow-up of six months in terms of complications. The mean age of the patients at the time of VP shunt placement was 3 months (Range: 8 days to 15 years). There were 46 boys $(76.6 \%)$ and 14 girls $(23.4 \%)$. The majority of the patients belonged to the 0-1-year age group $(76.8 \%)$ as shown in Figure 1.

The etiology of hydrocephalus is listed in Table 1.

Table 1. Causes of hydrocephalus

\begin{tabular}{lc}
\hline \multicolumn{1}{c}{ Cause } & Number \\
\hline Congenital aqueduct stenosis & $18(30.0 \%)$ \\
Congenital communicating HCP & $13(21.7 \%)$ \\
Associated with meningomyelocele & $11(18.3 \%)$ \\
Post meningitis & $6(10.0 \%)$ \\
Dandy-Walker malformation & $5(8.3 \%)$ \\
Tumors & $5(8.3 \%)$ \\
Others & $2(3.3 \%)$ \\
\hline
\end{tabular}




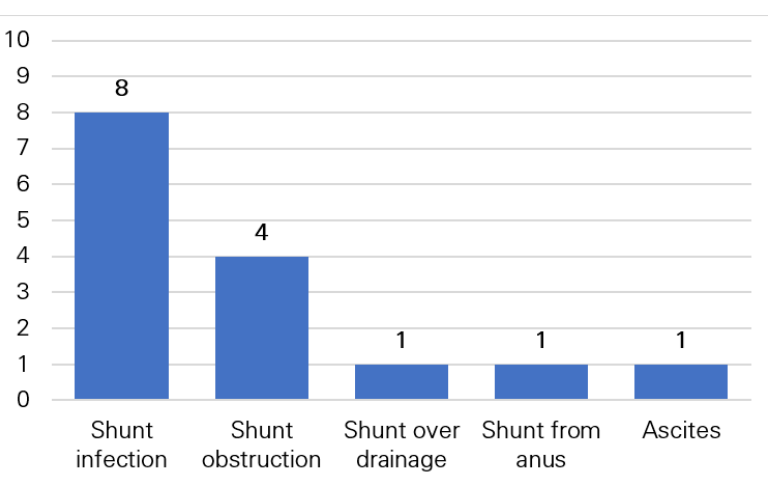

Fig 2. Bar diagram showing complications

Among them, congenital cause or associated with congenital anomaly accounted for over three fourth of total cases. The average duration of the procedure was 45 minutes. Most procedures were done on an emergency basis which accounted for $71.7 \%$. Fifty-two patients (86.7\%) underwent an anterior approach and eight patients (13.3\%) had a posterior approach for the placement of VP shunt. Fifteen patients $(25 \%)$ developed complications within six months follow-up period (Figure 2). The infective complications developed in eight patients $(13.3 \%)$ with four cases having culture positive. The most common organism isolated was Staphylococcus aureus. Among them, one patient developed ventriculitis (Figure 3) who underwent shunt exteriorization followed by an external ventricular drain. Four patients had mechanical complications in the form of shunt obstruction, three from proximal and one from distal end. One patient had shunt over drainage for which drainage of subdural collection, followed by upgrading of shunt chamber was done. One patient had ascites and another had shunt extrusion from anus without features of peritonitis. One patient $(1.67 \%)$ died during the study period who had ventriculitis.

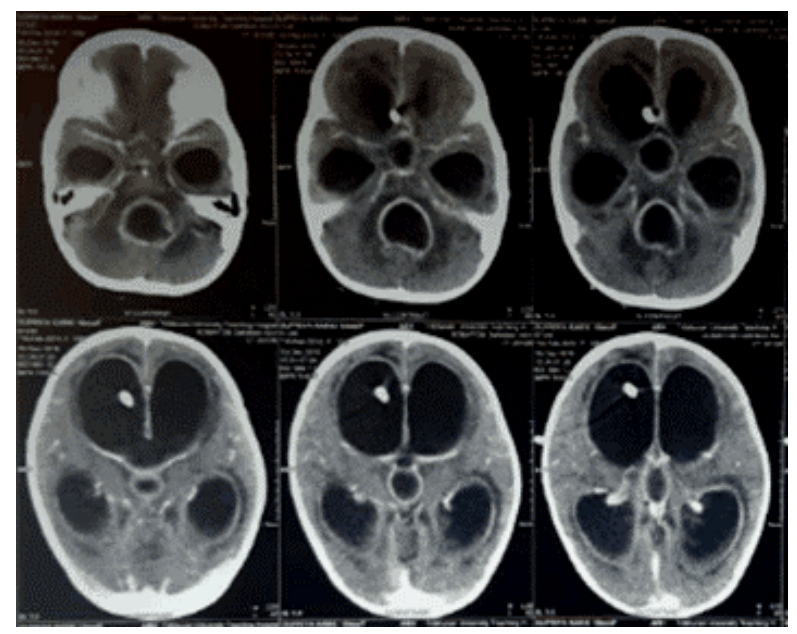

Fig 3. NCCT head showing features of ventriculitis

\section{DISCUSSION}

Placement of VP shunts for hydrocephalus is an established external CSF diversion procedure but with its own set of complications. In our study, $25 \%$ of patients had shunt- related complications. In the study by Karmacharya et al. and Dakurah et al. the reported shunt complications were $23.3 \%$ and $33.9 \%$ respectively. ${ }^{78}$ Similarly, Bawa $\mathrm{M}$ et al. the complication rate following VP shunt in congenital hydrocephalus was $32.01 \% .^{9}$

Overall shunt infection rate is $5 \%-8 \%$ in English literature..$^{10}$ In our study, it was $13.3 \%$ which is on the higher side. Similar findings were reported from Asian and African developing countries. Dakurah et al reported a shunt infection of $14.6 \%$; whereas Agarwal et al had a $15.6 \%$ shunt infection. ${ }^{8,6}$ As mentioned in the literature, the most common pathogen isolated is Staphylococcus epidermidis followed by Staphylococcus aureus. ${ }^{11}$ Different from literature, according to our culture sensitivity microbiological report, the most common microorganism was Staphylococcus aureus rather than epidermidis.

Shunt obstruction can occur anywhere along the shunt course. The incidence of shunt obstruction has been reported from $5 \%$ to $47 \% . .^{12}$ It was $10.94 \%$ as reported by $\operatorname{Pan~}^{\mathrm{P}^{13}}$ whereas in our study the shunt obstruction was $6.67 \%$.

In our study, all the patients who had shunt complications were shunted during the initial two years of age. Piatt and Carlson had increased shunt failures in patients less than two years of age. ${ }^{14}$ A similar observation was made by Liptak and McDonald in children less than one year of age..$^{15}$

The shunt-related mortality has been reported as being from $8.6 \%-13.7 \% .^{16}$ The shunt related mortality was $4.17 \%$ in the study by Agrawal et al. whereas, in our study, it was $1.67 \%$.

In 2007, Pirotte et al. reported no infections for 115 procedures performed using a strict protocol of limited implant and skin-edge manipulation, minimal personnel in the operating room, shunt surgery first in the morning, avoiding CSF leak, surgery duration less than 30 minutes, and systemic antibiotic prophylaxis. ${ }^{17}$ Similarly, Hydrocephalus Clinical Research Network (HCRN) centers reported an infection rate of $5.7 \%$ using the 11-step protocol in 2011.18 So these methods might be useful in reducing shunt related complications especially infection in our setup.

\section{CONCLUSION}

The shunt complications, including infection rate, were comparable with other studies while the mortality was less in our study. Shunt-related complications remain a major challenge in the field of neurosurgery. The shunt infection and obstruction 
still remain the most important complications. In spite of inherent problems, shunt surgery is the procedure of choice until a safer effective alternative is available. Large studies are required to know about long-term complications.

\section{CONFLICT OF INTEREST}

None declared.

\section{REFERENCES}

1. Rekate HL. The definition and classification of hydrocephalus: a personal recommendation to stimulate debate. Cerebrospinal Fluid Research. 2008;5:2

2. Dewan MC, Rattani A, Mekary $R$ et al. Global hydrocephalus epidemiology and incidence: systematic review and meta-analysis. Journal of Neurosurgery. 2018;1-15.

3. Kausch W. Die Behandlung des Hydrocephalus der Cleinen Kinder. Archiv Klinische Chirurgie. 1908;87:709-796.

4. Mukhida K, Sharma MR, Shilpakar SK. Management of Hydrocephalus with Ventriculoperitoneal Shunts: Review of 274 Cases. Nepal Journal of Neurosciences. 2004;1:104-112.

5. Kulkarni AV, JM Drake, M Lamberti-Pasculliet. Cerebrospinal fluid shunt infection: a prospective study of risk factors. Journal of Neurosurgery. 2001;94:195-201.

6. Agarwal N, Shukla RM, Agarwal D et al. Pediatric ventriculoperitoneal shunts and their complications: An analysis. Journal of Indian Association of Pediatric Surgeons. 2017;22:155157.

7. Karmacharya BG, Kumar P. A study on Complications of ventriculoperitoneal shunts surgery in Bir Hospital, Kathmandu, Nepal. Nepal Journal of Medical sciences. 2012;1:119-122.

8. Dakurah TK, Adams F, Iddrissu Metal. Management of Hydrocephalus with Ventriculoperitoneal Shunts: Review of 109 Cases of Children. World Neurosurgery. 2016;96:129-135.

9. Bawa M, Dash V, Mahalik S et al. Outcome Analysis of Patients of Congenital Hydrocephalus with Ventriculoperitoneal Shunt at a Tertiary Care Hospital in North India. Pediatric Neurosurgery. 2019;54:233-236.

10. Vinchon $M$, Rekate $H$, Kulkarni AV. Pediatric hydrocephalus outcomes: A review. Fluids and Barriers of the CNS. 2012;9:18.

11. Wong GK, Ip M, Poon WS et al. Antibiotics-impregnated ventricular catheter versus systemic antibiotics for prevention of nosocomial CSF and non-CSF infections: A prospective randomised clinical trial. Journal of Neurology, Neurosurgery, and Psychiatry. 2010;81:1064-1067.

12. Tyagi DK, Balasubramaniam S, Jayaswal SA et al. Outcome analysis of ventriculoperitoneal shunt procedures in hydrocephalus due to tubercular meningitis and non-infective cases. International Journal of Contemporary Pediatrics. 2016;3:12101215

13. Pan P. Outcome Analysis of Ventriculoperitoneal Shunt Surgery in Pediatric Hydrocephalus. Journal of Pediatric Neurosciences. 2018;13:176-181.

14. Piatt JH Jr., Carlson CV. A search for determinants of cerebrospinal fluid shunt survival: Retrospective analysis of a 14-year institutional experience. Pediatric Neurosurgery. 1993;19:233-241.

15. Liptak GS, McDonald JV. Ventriculoperitoneal shunts in children: Factors affecting shunt survival. Journal of Pediatric Neurosciences. 1986;12:289-293.

16. Tuli S, Tuli J, Drake J et al. Predictors of death in pediatric patients requiring cerebrospinal fluid shunts. Journal of Neurosurgery. 2004;100:442446.

17. Pirotte BJ, Lubansu A, Bruneau $M$ et al. Sterile surgical technique for shunt placement reduces the shunt infection rate in children: preliminary analysis of a prospective protocol in 115 consecutive procedures. Childs Nervous System. 2007; 23:1251-1261.

18. Kestle JR, Riva-Cambrin J, Wellons JC 3rd et al. A standardized protocol to reduce cerebrospinal fluid shunt infection: The hydrocephalus clinical research network quality improvement initiative. Journal of Neurosurgery Pediatrics. 2011;8:22-29 